\title{
An improved approach for studying oscillation of generalized Emden-Fowler neutral differential equation
}

\author{
Osama Moaaz ${ }^{1}$ Elmetwally M. Elabbasy ${ }^{1}$ and Belgees Qaraad ${ }^{1,2^{*}}$
}

\section{"Correspondence:}

belgeesmath2016@students.mans. edu.eg;

belgeesmath2016@gmail.com

'Department of Mathematics, Faculty of Science, Mansoura

University, Mansoura, Egypt 2 Department of Mathematics,

Faculty of Science, Saadah University, Saadah, Yemen

\section{Springer}

\begin{abstract}
The purpose of this work is to study the oscillation criteria for generalized Emden-Fowler neutral differential equation. We establish new oscillation criteria using both the technique of comparison with first order delay equations and the technique of Riccati transformation. Our new criteria are interesting as they improve, simplify, and complement some results that have been published recently in the literature. Moreover, we present an illustrating example.
\end{abstract}

MSC: $34 \mathrm{C} 10 ; 34 \mathrm{~K} 11$

Keywords: Generalized Emden-Fowler equation; Second-order; Neutral delay; Oscillation criteria

\section{Introduction}

In aeromechanical systems, where they have a significant role, in the theory of automatic control, in study of vibrating masses attached to an elastic bar (as the Euler equation), in the networks that have lossless transmission lines (as is the case in high-speed computers), and other applications, delay or neutral differential equations can be seen in the modeling of the mentioned phenomena, see $[1,2,5,15]$. As a result of these applications, research groups including us still study the differential equations with delay. The theory of oscillation of delay differential equations comes at the forefront of topics that have received the attention of researchers in recent times, see [1-29]. In the last decade, there has been a research movement to improve and develop the oscillation criteria of solutions of second order differential equations with delay (see $[9,10])$, neutral (see $[3,7,13])$ and advanced $($ see $[4,13])$.

In this work, we present new oscillation criteria for second-order Emden-Fowler delay differential equations of neutral type

$$
\left(r\left(v^{\prime}\right)^{\alpha}\right)^{\prime}(t)+q(t) f(u(\sigma(t)))=0, \quad t \geq t_{0}
$$

where $v(t)=u(t)+p(t) u(\tau(t))$ and $\alpha$ is a ratio of odd positive integers. We also assume that $r \in C^{1}\left(\left[t_{0}, \infty\right),(0, \infty)\right), \tau, \sigma, p, q \in C^{1}\left(\left[t_{0}, \infty\right), \mathbb{R}\right), \sigma(t) \leq t, \tau(t) \leq t, 0 \leq p(t)<$

(c) The Author(s) 2020. This article is licensed under a Creative Commons Attribution 4.0 International License, which permits use, sharing, adaptation, distribution and reproduction in any medium or format, as long as you give appropriate credit to the original author(s) and the source, provide a link to the Creative Commons licence, and indicate if changes were made. The images or other third party material in this article are included in the article's Creative Commons licence, unless indicated otherwise in a credit line to the material. If material is not included in the article's Creative Commons licence and your intended use is not permitted by statutory regulation or exceeds the permitted use, you will need to obtain permission directly from the copyright holder. To view a copy of this licence, visit http://creativecommons.org/licenses/by/4.0/. 


$$
\begin{gathered}
\min \{\pi(t) / \pi(\tau(t)), 1\}, q(t) \geq 0, \lim _{t \rightarrow \infty} \tau(t)=\lim _{t \rightarrow \infty} \sigma(t)=\infty, \text { and } \\
\pi\left(t_{0}\right):=\int_{t_{0}}^{\infty} \frac{1}{r^{\frac{1}{\alpha}}(v)} \mathrm{d} v<\infty .
\end{gathered}
$$

For the function $f$, we suppose that $f \in C(\mathbb{R}, \mathbb{R})$ and satisfies the following condition:

$$
f(u)>k u^{\beta} \quad \text { for all } u \neq 0,
$$

where $k$ is a positive constant and $\beta$ is a quotient of odd positive integers.

A solution of (1.1) means $u \in C\left(\left[t_{0}, \infty\right),[0, \infty)\right)$ with $t_{a}=\min \left\{\tau\left(t_{b}\right), \sigma\left(t_{b}\right)\right\}$, for some $t_{b}>$ $t_{0}$, which satisfies the property $r\left(v^{\prime}\right)^{\alpha} \in C^{1}\left(\left[t_{a}, \infty\right),[0, \infty)\right)$ and satisfies $(1.1)$ on $\left[t_{b}, \infty\right)$. We consider the nontrivial solutions of (1.1) existing on some half-line $\left[t_{b}, \infty\right)$ and satisfying the condition

$$
\left\{|u(t)|: t_{c} \leq t<\infty\right\}>0 \text { for any } t_{c} \geq t_{b}
$$

If $u$ is neither positive nor negative eventually, then $u(t)$ is called oscillatory, or it will be nonoscillatory.

For canonical form (if $\eta\left(t_{0}\right)=\infty$ ), there have been some studies that consider the oscillation and nonoscillation criteria of solutions of (1.1), see for example [19, 24].

For noncanonical form (if $\eta\left(t_{0}\right)<\infty$ ), Liu et al. [18] got necessary and appropriate conditions that ensure all solutions of (1.1) can be oscillatory, or they can tend to zero, following the conditions $\lim _{t \rightarrow \infty} p(t)=C$,

$$
p^{\prime}(t) \geq 0 \quad \text { and } \quad \tau^{\prime}(t) \geq 0
$$

Furthermore, Saker [23] developed the results of [18] in the sense that they established the conditions that assure all the solutions of Eq. (1.1) are oscillatory. The results of both [23] as well as [18] follow an approach that does lead to two conditions, and they are requested (1.2).

Wu et al. [28] established some criteria of oscillation for the neutral equation

$$
\left(r(t)\left|v^{\prime}(t)\right|^{\alpha-1} v^{\prime}(t)\right)^{\prime}+q(t)|u(\sigma(t))|^{\beta-1} u(\sigma(t))=0,
$$

under conditions (1.2),

$$
r^{\prime}(t) \geq 0, \quad \text { and } \quad \sigma(t) \leq \tau(t)
$$

This work aims at developing the oscillation theory of second order quasi-linear equations with delay argument. The use of the technique of comparison with first order delay equations and the technique of Riccati transformation helps us to get two various conditions, ensuring oscillation of (1.1) without requiring (1.2). In this paper, in the first two theorems, we simplify results in $[18,23,28]$ and obtain new criteria for ensuring oscillation of (1.1) without checking the additional conditions. Our criteria complement and extend the 
results in [7, 8]. In [7, Theorem 2.2], Bohner et al. proved that equation (1.1) with $\alpha=\beta$ is oscillatory if

$$
W:=\limsup _{t \rightarrow \infty} \pi^{\alpha}(t) \int_{t_{0}}^{t} q(v)\left(1-p(v) \frac{\pi(\tau(\sigma(v)))}{\pi(\sigma(v))}\right)^{\alpha} \mathrm{d} v>1 .
$$

In our paper, Theorems 2.5 and 2.6 substantially improve Theorem 2.2 in [7, Theorem 2.2], when $W \leq 1$.

The next lemma collects two useful inequalities that can be found in [29].

Lemma 1.1 Let $\alpha$ be a ratio of two odd positive integers. Then

$$
D V-C V^{(\alpha+1) / \alpha} \leq \frac{\alpha^{\alpha}}{(\alpha+1)^{\alpha+1}} \frac{D^{\alpha+1}}{C^{\alpha}}, \quad C>0,
$$

and

$$
A^{(\alpha+1) / \alpha}-(A-B)^{(\alpha+1) / \alpha} \leq \frac{1}{\alpha} B^{1 / \alpha}[(1+\alpha) A-B], \quad \alpha \geq 1, A B \geq 0 .
$$

\section{Main results}

In this section, we shall establish new oscillation criteria for (1.1). Let us define

$$
\begin{aligned}
& Q(t):=q(t)\left(1-p(t) \frac{\pi(\tau(\sigma(t)))}{\pi(\sigma(t))}\right)^{\beta}, \\
& \widehat{Q}(t):=\left(\frac{k}{r(t)} \int_{t_{1}}^{t} Q(v) \mathrm{d} v\right)^{1 / \alpha},
\end{aligned}
$$

and

$$
\eta(t):= \begin{cases}1 & \text { if } \alpha=\beta, \\ a_{1} & \text { if } \alpha>\beta, \\ a_{2} \pi^{\beta-\alpha}(t) & \text { if } \alpha<\beta,\end{cases}
$$

where $t_{1} \in\left[t_{0}, \infty\right)$ and $a_{1}, a_{2}$ are any positive constants.

Lemma 2.1 Assume that $u$ is an eventually nonincreasing positive solution of (1.1). Then $v^{\beta-\alpha}(t) \geq \eta(t)$.

Proof Let $v$ be an eventually positive solution of $(1.1)$ and $v^{\prime}(t)<0$. Then we have the following cases:

In the case where $\alpha=\beta$, it is easy to see that $v^{\beta-\alpha}(t)=1$.

Let $\alpha>\beta$. Since $v(t)$ is a nonincreasing positive function, there exists $M_{1}>0$ such that $v(t) \leq M_{1}$, which implies that

$$
v^{\beta-\alpha}(t) \geq M_{1}^{\beta-\alpha}=a_{1} .
$$

In the case $\alpha<\beta$, by using the decreasing property of $r\left(v^{\prime}\right)^{\alpha}$, we obtain

$$
r(t)\left(v^{\prime}(t)\right)^{\alpha} \leq r\left(t_{1}\right)\left(v^{\prime}\left(t_{1}\right)\right)^{\alpha}=-M_{2}<0,
$$


hence

$$
v^{\prime}(t) \leq\left(\frac{-M_{2}}{r(t)}\right)^{\frac{1}{\alpha}} .
$$

Integrating the last inequality from $t$ to $\infty$, we get

$$
-v(t) \leq-M_{2}^{\frac{1}{\alpha}} \pi(t) .
$$

Thus, we include that

$$
v^{\beta-\alpha}(t) \geq M_{2}^{\frac{\beta-\alpha}{\alpha}} \pi^{\beta-\alpha}(t)=a_{2} \pi^{\beta-\alpha}(t) .
$$

Therefore, we have $v^{\beta-\alpha}(t) \geq \eta(t)$. The proof of the lemma is complete.

Lemma 2.2 Let $u$ be a positive solution of $(1.1)$ on $\left[t_{0}, \infty\right)$. If

$$
\int_{t_{1}}^{\infty} Q(v) \mathrm{d} v=\infty
$$

for $t_{1} \geq t_{0}$, then

$(\mathbf{H}) v$ is decreasing, $r\left(v^{\prime}\right)^{\alpha}$ is nonincreasing, eventually.

Proof Let $u$ be a positive solution of (1.1) on $\left[t_{0}, \infty\right)$. Then we suppose that there exists $t_{1} \in\left[t_{0}, \infty\right)$ such that $u(t)>0, u(\tau(t))>0$, and $u(\sigma(t))>0$ for all $t \in\left[t_{1}, \infty\right)$. Obviously, we find $v(t) \geq u(t)$ and

$$
\left(r\left(v^{\prime}\right)^{\alpha}\right)^{\prime}(t)=-q(t) f(u(\sigma(t))) \leq 0 .
$$

Therefore, $v^{\prime}$ is either eventually negative or eventually positive.

Suppose now that $v^{\prime}>0$ on $\left[t_{1}, \infty\right)$. Then $u(t) \geq(1-p(t)) v(t)$, and (2.2) becomes

$$
\left(r\left(v^{\prime}\right)^{\alpha}\right)^{\prime}(t) \leq-k q(t)(1-p(\sigma(t)))^{\beta} v^{\beta}(\sigma(t)) .
$$

Since $\pi(\tau(\sigma(t))) \geq \pi(\sigma(t))$, we get

$$
1-p(\sigma(t)) \geq 1-p(\sigma(t)) \frac{\pi(\tau(\sigma(t)))}{\pi(\sigma(t))} .
$$

Integrating (2.3) from $t_{1}$ to $t$ and using (2.4), we get

$$
\begin{aligned}
r(t)\left(v^{\prime}(t)\right)^{\alpha} & \leq r\left(t_{1}\right)\left(v^{\prime}\left(t_{1}\right)\right)^{\alpha}-k \int_{t_{1}}^{t} q(v)(1-p(\sigma(v)))^{\beta} v^{\beta}(\sigma(t)) \mathrm{d} v \\
& \leq r\left(t_{1}\right)\left(v^{\prime}\left(t_{1}\right)\right)^{\alpha}-k v^{\beta}\left(\sigma\left(t_{1}\right)\right) \int_{t_{1}}^{t} q(v)(1-p(\sigma(v)))^{\beta} \mathrm{d} v \\
& \leq r\left(t_{1}\right)\left(v^{\prime}\left(t_{1}\right)\right)^{\alpha}-k v^{\beta}\left(\sigma\left(t_{1}\right)\right) \int_{t_{1}}^{t} Q(v) \mathrm{d} v,
\end{aligned}
$$

a contradiction with positivity of $v^{\prime}(t)$. The proof of this lemma is complete. 
Lemma 2.3 Let $u$ be a positive solution of $(1.1)$ on $\left[t_{0}, \infty\right)$. If

$$
\int_{t_{1}}^{\infty}\left(\frac{1}{r(s)} \int_{t_{2}}^{s} Q(v) \mathrm{d} v\right)^{1 / \alpha} \mathrm{d} s=\infty
$$

for $t_{1} \geq t_{0}$, then $(\mathbf{H})$ holds and

$$
\lim _{t \rightarrow \infty} v(t)=\infty
$$

Proof Let $u$ be a positive solution of (1.1) on $\left[t_{0}, \infty\right)$. From $\pi\left(t_{0}\right)<\infty$ and (2.5), we have that (2.1) holds. Hence, from Lemma 2.2, we have that $v^{\prime}(t)<0,(2.3)$ and (2.4) hold.

Now, since $v>0$ and $v^{\prime}<0$, we get that $\lim _{t \rightarrow \infty} v(t)=c \geq 0$. Suppose that $c>0$. Then there exists $t_{2} \geq t_{1}$ such that $v(\sigma(t)) \leq c$. From (2.3) and (2.4), we obtain

$$
\left(r\left(v^{\prime}\right)^{\alpha}\right)^{\prime}(t) \leq-k c^{\beta} Q(t)
$$

for $t \geq t_{2}$. Integrating two times this inequality from $t_{2}$ to $t$, we get, after the first integration,

$$
v^{\prime}(t) \leq-\left(k c^{\beta}\right)^{1 / \alpha}\left(\frac{1}{r(t)} \int_{t_{2}}^{t} Q(s) \mathrm{d} s\right)^{1 / \alpha} .
$$

After the second integration, we obtain

$$
v(t)-v\left(t_{2}\right) \leq-\left(k c^{\beta}\right)^{1 / \alpha} \int_{t_{2}}^{t}\left(\frac{1}{r(s)} \int_{t_{2}}^{s} Q(v) \mathrm{d} v\right)^{1 / \alpha} \mathrm{d} s .
$$

This implies that $\lim _{t \rightarrow \infty} v(t)=-\infty$, which contradicts $v>0$. The proof of this lemma is complete.

Theorem 2.1 If

$$
\int_{t_{1}}^{\infty}\left(\frac{1}{r(t)} \int_{t_{1}}^{t} Q(v) \pi^{\beta}(\sigma(v)) \mathrm{d} v\right)^{1 / \alpha} \mathrm{d} t=\infty
$$

for $t_{1} \geq t_{0}$, then (1.1) is oscillatory.

Proof Let $u$ be a positive solution of (1.1) on $\left[t_{0}, \infty\right)$ (assume the converse). Then we suppose that there exists $t_{1} \in\left[t_{0}, \infty\right)$ such that $u(t)>0, u(\tau(t))>0$ and $u(\sigma(t))>0$ for all $t \in\left[t_{1}, \infty\right)$. Since $\pi\left(t_{0}\right)<\infty$ and (2.7), we have that $\int_{t_{1}}^{t} Q(v) \pi^{\beta}(\sigma(v)) \mathrm{d} v$ must be unbounded. Thus, and from the fact $\pi^{\prime}(t)<0$, it is easy to see that (2.1) holds. Hence, from Lemma 2.2, we have that $v^{\prime}(t)<0$ and (2.2) holds. Since

$$
v(t) \geq-\int_{t}^{\infty} v^{\prime}(v) \mathrm{d} v=-\int_{t}^{\infty} \frac{r^{1 / \alpha}(v) v^{\prime}(v)}{r^{1 / \alpha}(v)} \mathrm{d} v \geq-\pi(t) r^{1 / \alpha}(t) v^{\prime}(t)
$$

it follows that

$$
\frac{\mathrm{d}}{\mathrm{d} t}\left(\frac{v(t)}{\pi(t)}\right) \geq 0
$$


In view of the definition of $v$, we deduce

$$
\begin{aligned}
u(t) & =v(t)-p(t)(u(\tau(t))) \geq v(t)-p(t)(v(\tau(t))) \\
& \geq v(t)\left(1-p(t) \frac{\pi(\tau(t))}{\pi(t)}\right) .
\end{aligned}
$$

Consequently, (2.2) becomes

$$
\begin{aligned}
\left(r\left(v^{\prime}\right)^{\alpha}\right)^{\prime}(t) & \leq-k q(t)\left(1-p(t) \frac{\pi(\tau(\sigma(t)))}{\pi(\sigma(t))}\right)^{\beta} v^{\beta}(\sigma(t)) \\
& =-k Q(t) v^{\beta}(\sigma(t)) .
\end{aligned}
$$

From the monotonicity property of $r(t)\left(v^{\prime}(t)\right)^{\alpha}$, we have

$$
-r(t)\left(v^{\prime}(t)\right)^{\alpha} \geq-r\left(t_{1}\right)\left(v^{\prime}\left(t_{1}\right)\right)^{\alpha}=: L>0,
$$

which in view of (2.8) implies

$$
v^{\beta}(t) \geq L^{\frac{\beta}{\alpha}} \pi^{\beta}(t) .
$$

From (2.10), (2.9) becomes

$$
\left(r\left(v^{\prime}\right)^{\alpha}\right)^{\prime}(t) \leq-k L^{\frac{\beta}{\alpha}} Q(t) \pi^{\beta}(\sigma(t)) .
$$

Integrating (2.11) from $t_{1}$ to $t$, we obtain

$$
\begin{aligned}
r(t)\left(v^{\prime}(t)\right)^{\alpha} & \leq r\left(t_{1}\right)\left(v^{\prime}\left(t_{1}\right)\right)^{\alpha}-k L^{\frac{\beta}{\alpha}} \int_{t_{1}}^{t} Q(v) \pi^{\beta}(\sigma(v)) \mathrm{d} v \\
& \leq-k L^{\frac{\beta}{\alpha}} \int_{t_{1}}^{t} Q(v) \pi^{\beta}(\sigma(v)) \mathrm{d} v .
\end{aligned}
$$

Integrating (2.12) from $t_{1}$ to $t$ and using (2.7), we get

$$
v(t) \leq v\left(t_{1}\right)-k^{\frac{1}{\alpha}} L^{\beta} \int_{t_{1}}^{t}\left(\frac{1}{r(v)} \int_{t_{1}}^{v} Q(v) \pi^{\beta}(\sigma(v)) \mathrm{d} v\right)^{\frac{1}{\alpha}} \mathrm{d} v,
$$

which in view of (2.7) contradicts the positivity of $v(t)$. The proof of the theorem is complete.

Theorem 2.2 Assume that $\sigma^{\prime}(t)>0$. If

$$
\limsup _{t \rightarrow \infty} \pi^{\alpha}(t) \eta(t) \int_{t_{1}}^{t} Q(v) \mathrm{d} v>1
$$

for $t_{1} \geq t_{0}$, then (1.1) is oscillatory. 
Proof To the contrary, we suppose that $u$ is a positive solution of $(1.1)$ on $\left[t_{0}, \infty\right)$. Then there exists $t_{1} \geq t_{0}$ such that $u(\tau(t))>0$ and $u(\sigma(t))>0$ for all $t \geq t_{1}$. From (2.13) and $\pi\left(t_{0}\right)<\infty$, we get $(2.1)$ holds. Using Lemma 2.2, we get that $v^{\prime}<0$ on $\left[t_{1}, \infty\right)$. As in the proof of Theorem 2.1, we get (2.8) and (2.9) hold. By integrating (2.9) from $t_{1}$ to $t$, we get

$$
\begin{aligned}
r(t)\left(v^{\prime}(t)\right)^{\alpha} & \leq r\left(t_{1}\right)\left(v^{\prime}\left(t_{1}\right)\right)^{\alpha}-k \int_{t_{1}}^{t} Q(v) v^{\beta}(\sigma(v)) \mathrm{d} v \\
& \leq-k v^{\beta}(\sigma(t)) \int_{t_{1}}^{t} Q(v) \mathrm{d} v .
\end{aligned}
$$

Since $\sigma(t) \leq t$ and $v^{\prime}(t)<0$, we obtain

$$
r(t)\left(v^{\prime}(t)\right)^{\alpha} \leq-k v^{\alpha}(t) v^{\beta-\alpha}(t) \int_{t_{1}}^{t} Q(v) \mathrm{d} v .
$$

By Lemma 2.1 and (2.8) we arrive at

$$
-r(t)\left(v^{\prime}(t)\right)^{\alpha} \geq-r(t)\left(v^{\prime}(t)\right)^{\alpha} \pi^{\alpha}(t) \eta(t) \int_{t_{1}}^{t} Q(v) \mathrm{d} v,
$$

and so

$$
\pi^{\alpha}(t) \eta(t) \int_{t_{1}}^{t} Q(v) \mathrm{d} v \leq 1
$$

a contradiction with (2.13). Then the proof is complete.

Theorem 2.3 Assume that (2.1) holds. If the first order delay differential equation

$$
v^{\prime}(t)+\widehat{Q}(t) v^{\beta / \alpha}(\sigma(t))=0
$$

is oscillatory, then (1.1) is oscillatory.

Proof To the contrary, we suppose that $u$ is a positive solution of $(1.1)$ on $\left[t_{0}, \infty\right)$. Then there exists $t_{1} \geq t_{0}$ such that $u(\tau(t))>0$ and $u(\sigma(t))>0$ for all $t \geq t_{1}$. Using (2.1) and Lemma 2.2, we get that $v^{\prime}<0$ on $\left[t_{1}, \infty\right)$. As in the proof of Theorem 2.2, we get (2.14) holds. From (2.14), it is clear that $v$ is a positive solution of the first order differential inequality

$$
v^{\prime}(t)+\widehat{Q}(t) v^{\beta / \alpha}(\sigma(t)) \leq 0 .
$$

In view of [25, Lemma 1], we see that the first-order delay differential equation (2.16) has a positive solution, a contradiction. Then the proof is complete.

Corollary 2.1 Assume that $\alpha=\beta$. If

$$
\liminf _{t \rightarrow \infty} \int_{\sigma(t)}^{t} \widehat{Q}(v) \mathrm{d} v>\frac{1}{\mathrm{e}}
$$

then (1.1) is oscillatory. 
Proof In view of [16, Theorem 2], condition (2.17) implies oscillation of (2.16). On the other hand, if suffices to note that

$$
\int_{t_{0}}^{\infty} \widehat{Q}(v) \mathrm{d} v=\infty
$$

is necessary for the validity of (2.1). Therefore, the proof is complete.

Corollary 2.2 Assume that $\alpha>\beta>0$. If

$$
\int_{t_{0}}^{\infty} \widehat{Q}(v) d v=\infty
$$

then (1.1) is oscillatory.

Proof Since $\beta / \alpha \in(0,1)$, it is shown that all the solutions of (2.16) oscillate if and only if (2.18) holds, see [12] and [17]. On the other hand, we see that (2.18) is necessary for the validity of (2.1). Therefore, the proof is complete.

Corollary 2.3 Assume that $\alpha<\beta, \sigma(t)$ is continuously differentiable, $\sigma^{\prime}(t)>0$ and (2.1) holds. If there exists a continuously differentiable function $\xi(t)$ such that $\xi^{\prime}(t)>0$, $\lim _{t \rightarrow \infty} \xi(t)=\infty$,

$$
\limsup _{t \rightarrow \infty} \frac{\beta \xi^{\prime}(\sigma(t)) \sigma^{\prime}(t)}{\alpha \xi^{\prime}(t)}<1
$$

and

$$
\liminf _{t \rightarrow \infty}\left[\frac{\widehat{Q}(t)}{\xi^{\prime}(t)} e^{-\xi(t)}\right]>0,
$$

then (1.1) is oscillatory.

Proof In view of [25, Theorem 1], condition (2.19) implies oscillation of (2.16).

The following results serve as an improvement of Theorems 2.2, when $\alpha=\beta$ and

$$
\limsup _{t \rightarrow \infty} \pi^{\alpha}(t) \eta(t) \int_{t_{1}}^{t} Q(v) \mathrm{d} v \leq 1 .
$$

For the simplicity, we define the following notations:

$$
m:=\liminf _{t \rightarrow \infty} \frac{k}{\pi(t)} \int_{t}^{\infty} \pi^{\alpha+1}(s) Q(s) \mathrm{d} s
$$

and

$$
M:=\limsup _{t \rightarrow \infty} \pi(t)\left(k \int_{t_{0}}^{t} Q(s) \mathrm{d} s\right)^{1 / \alpha} .
$$


Theorem 2.4 Assume that $\alpha=\beta$ and (2.5) is satisfied. If

$m>\alpha$

or

$$
m \leq \alpha \quad \text { and } \quad M>1-\frac{m}{\alpha}
$$

then (1.1) is oscillatory.

Proof Suppose against the assumption of theorem that equation (1.1) has a nonoscillatory solution $u$ on $\left[t_{0}, \infty\right)$. Without loss of generality, we may assume that $u(t)>0$ and $u(\sigma(t))>$ 0 for $t \geq t_{1} \geq t_{0}$. Let

$$
g^{\prime}(t)=\left(v(t)+r^{1 / \alpha}(t) v^{\prime}(t) \pi(t)\right)^{\prime}=\pi(t)\left(r^{1 / \alpha}(t) v^{\prime}(t)\right)^{\prime},
$$

then

$$
\left(\left(r^{1 / \alpha}(t) v^{\prime}(t)\right)^{\alpha}\right)^{\prime}=\alpha\left(r^{1 / \alpha}(t) v^{\prime}(t)\right)^{\alpha-1}\left(r^{1 / \alpha}(t) v^{\prime}(t)\right)^{\prime}
$$

Combining (2.22) and (2.23), and using inequality (2.9), we get

$$
\begin{aligned}
g^{\prime}(t) & =\frac{1}{\alpha} \pi(t)\left(r^{1 / \alpha}(t) v^{\prime}(t)\right)^{1-\alpha}\left(\left(r(t)\left(v^{\prime}(t)\right)^{\alpha}\right)\right)^{\prime} \\
& \leq-\frac{k}{\alpha} \pi(t)\left(r^{1 / \alpha}(t) v^{\prime}(t)\right)^{1-\alpha} Q(t) v^{\alpha}(\sigma(t)) .
\end{aligned}
$$

Integrating (2.24) from $t$ to $\infty$, we get

$$
\begin{aligned}
g(t) & \geq \frac{k}{\alpha} \int_{t}^{\infty} \pi(s) Q(s)\left(r^{1 / \alpha}(s) v^{\prime}(s)\right)^{1-\alpha} v^{\alpha}(s) \mathrm{d} s \\
& \geq-\frac{k}{\alpha} \int_{t}^{\infty} \pi(t) Q(s)\left(r^{\frac{1}{\alpha}}(s) v^{\prime}(s)\right)^{1-\alpha}\left(-\pi(t) r^{1 / \alpha}(s) v^{\prime}(s)\right)^{\alpha-1} v(s) \mathrm{d} s \\
& \geq \frac{k}{\alpha} \frac{v(t)}{\pi(t)} \int_{t}^{\infty} \pi^{\alpha+1}(s) Q(s) \mathrm{d} s .
\end{aligned}
$$

It follows that

$$
v(t)+r^{1 / \alpha}(t) v^{\prime}(t) \pi(t) \geq \frac{k}{\alpha} \frac{v(t)}{\pi(t)} \int_{t}^{\infty} \pi^{\alpha+1}(s) Q(s) \mathrm{d} s,
$$

and so

$$
v(t)\left(1-\frac{k}{\alpha} \frac{1}{\pi(t)} \int_{t}^{\infty} \pi^{\alpha+1}(s) Q(s) \mathrm{d} s\right) \geq-r^{1 / \alpha}(t) v^{\prime}(t) \pi(t)>0 .
$$

Now, let (2.20) hold. It follows from (2.20) that there exists $\epsilon>0$ such that

$$
m-\epsilon>\alpha \text {. }
$$


By virtue of definition of $m$, we see that

$$
1-\frac{k}{\alpha} \frac{1}{\pi(t)} \int_{t}^{\infty} \pi^{\alpha+1}(s) Q(s) \mathrm{d} s \leq 1-\frac{1}{\alpha}(m-\epsilon)<0,
$$

this contradicts the positivity of $v$.

Assume next that the case $m \leq \alpha$ holds. Proceeding as in the proof of Theorem 2.2, we get (2.14). Thus, by (2.25), we get

$$
\begin{aligned}
& -r^{1 / \alpha}(t)\left(v^{\prime}(t)\right)\left(1-\frac{k}{\alpha \pi(t)} \int_{t}^{\infty} \pi^{\alpha+1}(s) Q(s) \mathrm{d} s\right) \\
& \geq k v(t)\left(1-\frac{k}{\alpha \pi(t)} \int_{t}^{\infty} \pi^{\alpha+1}(s) Q(s) \mathrm{d} s\right)\left(\int_{t_{0}}^{t} Q(s) \mathrm{d} s\right)^{1 / \alpha} \\
& \geq-k r^{1 / \alpha}(t) v^{\prime}(t) \pi(t)\left(\int_{t_{0}}^{t} Q(s) \mathrm{d} s\right)^{1 / \alpha},
\end{aligned}
$$

that is,

$$
\left(1-\frac{k}{\alpha \pi(t)} \int_{t}^{\infty} \pi^{\alpha+1}(s) Q(s) \mathrm{d} s\right) \geq k \pi(t)\left(\int_{t_{0}}^{t} Q(s) \mathrm{d} s\right)^{\frac{1}{\alpha}}
$$

Hence,

$$
\limsup _{t \rightarrow \infty} \pi(t)\left(k \int_{t_{0}}^{t} Q(s) \mathrm{d} s\right)^{\frac{1}{\alpha}} \leq 1-\liminf _{t \rightarrow \infty} \frac{k}{\alpha \pi(t)} \int_{t}^{\infty} \pi^{\alpha+1}(s) Q(s) \mathrm{d} s
$$

which implies

$$
M \leq \frac{\alpha-m}{\alpha}
$$

this contradicts (2.21). Then the proof is complete.

Lemma 2.4 Assume that (1.1) has an eventually positive solution $u$ on $\left[t_{0}, \infty\right)$. Then there exist $T \geq t_{1}$ and $\epsilon>0$ such that

$$
\left(\frac{v}{\pi^{N}}\right) \text { is nonincreasing on }[T, \infty) \text {, }
$$

where $N=M-\epsilon$.

Proof Assume that $u$ is a positive solution of $(1.1)$ on $[T, \infty)$. By Lemma 2.3, $u(t)$ satisfies $(\mathbf{H})$ and (2.6). Proceeding as in the proof of Theorem 2.2, we have (2.14) holds. Now, we see that

$$
\begin{aligned}
\frac{\mathrm{d}}{\mathrm{d} t}\left(\frac{v(t)}{\pi^{N}(t)}\right) & =\frac{r^{1 / \alpha}(t) v^{\prime}(t) \pi^{N}(t)+N v(t) \pi^{N-1}(t)}{r^{1 / \alpha}(t) \pi^{2 N}(t)} \\
& \leq \frac{v(t)}{r^{1 / \alpha}(t) \pi^{N+1}(t)}\left(N+\frac{r^{1 / \alpha}(t) v^{\prime}(t) \pi(t)}{v(t)}\right) .
\end{aligned}
$$


In view of (2.14), we note that

$$
\frac{-r^{1 / \alpha}(t) v^{\prime}(t)}{v(t)} \geq\left(k \int_{t_{0}}^{t} Q(s) \mathrm{d} s\right)^{1 / \alpha}
$$

which implies

$$
N+\frac{r^{1 / \alpha}(t) v^{\prime}(t) \pi(t)}{v(t)} \leq N-\pi(t)\left(k \int_{t_{0}}^{t} Q(s) \mathrm{d} s\right)^{1 / \alpha}<0
$$

Therefore, (2.27) becomes

$$
\frac{\mathrm{d}}{\mathrm{d} t}\left(\frac{v(t)}{\pi^{N}(t)}\right)<0
$$

Then the proof is complete.

Theorem 2.5 Assume that $\alpha=\beta$ and (2.5) holds. If there is a constant $\kappa$ such that

$$
\frac{\pi(\sigma(t))}{\pi(t)} \geq \kappa>1
$$

for all $t \geq t_{0}$ and

$$
M \kappa^{M}>1
$$

then (1.1) is oscillatory.

Proof Proceeding as in the proof of Theorem 2.2, we get that (2.14) holds. By Lemma 2.4, we see that

$$
v(\sigma(t)) \geq v(t) \kappa^{N}
$$

From (2.14), we get

$$
-r(t)\left(v^{\prime}(t)\right)^{\alpha} \geq k v^{\alpha}(t) \kappa^{\alpha N} \int_{t_{0}}^{t} Q(s) \mathrm{d} s .
$$

In view of (2.8), we obtain

$$
-r(t)\left(v^{\prime}(t)\right)^{\alpha} \geq-k r(t)\left(v^{\prime}(t)\right)^{\alpha} \pi^{\alpha}(t) \kappa^{\alpha N} \int_{t_{0}}^{t} Q(s) \mathrm{d} s .
$$

It follows that

$$
1 \geq \kappa^{N} \pi(t)\left(k \int_{t_{0}}^{t} Q(s) \mathrm{d} s\right)^{1 / \alpha} .
$$

Taking the lim sup on both sides, we obtain a contradiction. Then the proof is complete. 
Theorem 2.6 Assume that $\alpha=\beta$, (2.5) holds, and there is a constant $\kappa$ such that (2.28) holds. If (2.20) or

$$
m \leq \alpha \quad \text { and } \quad M \kappa^{M}>1-\frac{m}{\alpha}
$$

then (1.1) is oscillatory.

Proof As in the proof of Theorem 2.4, if we replace (2.14) by (2.31), then we get

$$
\begin{aligned}
& -r^{1 / \alpha}(t)\left(v^{\prime}(t)\right)\left(1-\frac{k}{\alpha \pi(t)} \int_{t}^{\infty} \pi^{\alpha+1}(s) Q(s) \mathrm{d} s\right) \\
& \geq v(t) \kappa^{N}\left(1-\frac{k}{\alpha \pi(t)} \int_{t}^{\infty} \pi^{\alpha+1}(s) Q(s) \mathrm{d} s\right)\left(k \int_{t_{0}}^{t} Q(s) \mathrm{d} s\right)^{1 / \alpha}
\end{aligned}
$$

and so

$$
\left(1-\frac{k}{\alpha \pi(t)} \int_{t}^{\infty} \pi^{\alpha+1}(s) Q(s) \mathrm{d} s\right) \geq \pi(t) \kappa^{N}\left(k \int_{t_{0}}^{t} Q(s) \mathrm{d} s\right)^{\frac{1}{\alpha}}
$$

Taking lim sup on both sides, we obtain

$$
\limsup _{t \rightarrow \infty} \pi(t) \kappa^{N}\left(k \int_{t_{0}}^{t} Q(s) \mathrm{d} s\right)^{\frac{1}{\alpha}} \leq 1-\liminf _{t \rightarrow \infty} \frac{k}{\alpha \pi(t)} \int_{t}^{\infty} \pi^{\alpha+1}(s) Q(s) \mathrm{d} s
$$

Therefore,

$$
\kappa^{N} M \leq 1-\frac{m}{\alpha} .
$$

Then the proof is complete.

In the next theorems, by using a generalized Riccati substitution, we establish new oscillation criteria of (1.1).

Theorem 2.7 Assume that $\sigma^{\prime}(t)>0$ and $\alpha \geq 1$. If there exist functions $\delta, \varphi \in C^{1}\left(\left[t_{0}, \infty\right)\right.$, $(0, \infty))$ such that

$$
\limsup _{t \rightarrow \infty} \int_{t_{0}}^{t}\left(\Psi(v)-\frac{\delta(v) r(v)\left(\Phi_{+}(v)\right)^{\alpha+1}}{(\alpha+1)^{\alpha+1}}\right) \mathrm{d} v=\infty
$$

and

$$
\limsup _{t \rightarrow \infty} \int_{t_{0}}^{t}\left(\varphi(v) G(v)-\frac{r(\sigma(v))\left(\varphi_{+}^{\prime}(v)\right)^{\alpha+1}}{(\alpha+1)^{\alpha+1}\left(\varphi(v) \sigma^{\prime}(v)\right)^{\alpha}}\right) \mathrm{d} v=\infty,
$$

where

$$
\Phi(t):=\frac{\delta^{\prime}(t)}{\delta(t)}+\frac{1+\alpha}{r^{1 / \alpha}(t) \pi(t)},
$$




$$
\begin{aligned}
& \Psi(t):=\delta(t)\left(k \eta(t) Q(t)+\frac{1-\alpha}{r^{1 / \alpha}(t) \pi^{\alpha+1}(t)}\right), \\
& G(t):=k \eta(\sigma(t)) q(t)(1-p(\sigma(t)))^{\beta}
\end{aligned}
$$

and $H_{+}(t)=\max \{H(t), 0\}$, then $(1.1)$ is oscillatory.

Proof To the contrary, we suppose that $u$ is a positive solution of $(1.1)$ on $\left[t_{0}, \infty\right)$. Thus, there exists $t_{1} \geq t_{0}$ such that $u(\tau(t))>0$ and $u(\sigma(t))>0$ for all $t \geq t_{1}$. Then we get that $v^{\prime}$ has one sign eventually.

Now, we let $v^{\prime}(t)<0$ for $t \geq t_{1}$. As in the proof of Theorem 2.1, we get (2.9) holds. Define the function $\omega(t)$ by

$$
\omega(t)=\delta(t)\left[\frac{r(t)\left(v^{\prime}(t)\right)^{\alpha}}{v^{\alpha}(t)}+\frac{1}{\pi^{\alpha}(t)}\right] .
$$

From (2.8), we see that $\omega(t) \geq 0$. By differentiating (2.34), we get

$$
\begin{aligned}
\omega^{\prime}(t)= & \frac{\delta^{\prime}(t)}{\delta(t)} \omega(t)+\delta(t) \frac{\left(r(t)\left(v^{\prime}(t)\right)^{\alpha}\right)^{\prime}}{v^{\alpha}(t)}-\alpha \delta(t) r(t)\left(\frac{v^{\prime}(t)}{v(t)}\right)^{\alpha+1}+\frac{\alpha \delta(t)}{r^{\frac{1}{\alpha}}(t) \pi^{\alpha+1}(t)} \\
= & \frac{\delta^{\prime}(t)}{\delta(t)} \omega(t)+\delta(t) \frac{\left(r(t)\left(v^{\prime}(t)\right)^{\alpha}\right)^{\prime}}{v^{\alpha}(t)}+\frac{\alpha \delta(t)}{r^{\frac{1}{\alpha}}(t) \pi^{\alpha+1}(t)} \\
& -\alpha \delta(t) r(t)\left(\frac{\omega(t)}{\delta(t) r(t)}-\frac{1}{r(t) \pi^{\alpha}(t)}\right)^{(\alpha+1) / \alpha} .
\end{aligned}
$$

Using inequality (1.6) with

$$
A:=\frac{\omega(t)}{\delta(t) r(t)} \quad \text { and } \quad B:=\frac{1}{r(t) \pi^{\alpha}(t)},
$$

we obtain

$$
\begin{aligned}
{\left[\frac{\omega(t)}{\delta(t) r(t)}-\frac{1}{r(t) \pi^{\alpha}(t)}\right]^{\frac{\alpha+1}{\alpha}} \geq } & \left(\frac{\omega(t)}{\delta(t) r(t)}\right)^{\frac{\alpha+1}{\alpha}} \\
& -\frac{1}{\alpha r(t)^{\frac{1}{\alpha}} \pi(t)}\left[\frac{(\alpha+1) \omega(t)}{\delta(t) r(t)}-\frac{1}{r(t) \pi^{\alpha}(t)}\right] .
\end{aligned}
$$

Using Lemma 2.1 with (2.9), we have

$$
\frac{\left(r(t)\left(v^{\prime}(t)\right)^{\alpha}\right)^{\prime}}{v^{\alpha}(t)} \leq-k Q(t) \frac{v^{\beta}(\sigma(t))}{v^{\alpha}(t)} \leq-k \eta(t) Q(t) .
$$

From (2.35)-(2.37), we find

$$
\begin{aligned}
\omega^{\prime}(t) \leq & \frac{\delta^{\prime}(t)}{\delta(t)} \omega(t)-k \delta(t) \eta(t) Q(t)-\alpha \delta(t) r(t)\left(\left(\frac{\omega(t)}{\delta(t) r(t)}\right)^{\frac{\alpha+1}{\alpha}}\right. \\
& \left.-\frac{1}{\alpha r(t)^{\frac{1}{\alpha}} \pi(t)}\left[(\alpha+1) \frac{\omega(t)}{\delta(t) r(t)}-\frac{1}{r(t) \pi^{\alpha}(t)}\right]\right)+\frac{\alpha \delta(t)}{r^{\frac{1}{\alpha}}(t) \pi^{\alpha+1}(t)} \\
= & \Phi(t) \omega(t)-\Psi(t)-\alpha \frac{1}{(\delta(t) r(t))^{\frac{1}{\alpha}}} \omega(t)^{\frac{\alpha+1}{\alpha}}
\end{aligned}
$$


By inequality 1.5 with $C:=\alpha(\delta(t) r(t))^{-1 / \alpha}, D:=\Phi(t)$, and $V:=\omega(t)$, we obtain

$$
\omega^{\prime}(t) \leq-\Psi(t)+\frac{\delta(t) r(t)(\Phi(t))^{\alpha+1}}{(\alpha+1)^{\alpha+1}}
$$

Integrating from $t_{2}$ to $t$, we obtain

$$
\int_{t_{2}}^{t}\left(\Psi(v)-\frac{\delta(v) r(v)\left(\Phi_{+}(v)\right)^{\alpha+1}}{(\alpha+1)^{\alpha+1}}\right) \mathrm{d} v \leq \omega\left(t_{2}\right)-\omega(t) \leq \omega\left(t_{2}\right),
$$

which contradicts (2.32).

On the other hand, let $v^{\prime}(t)>0$ for all $t \geq t_{2}$. It is easy to prove that $u(t) \geq(1-p(t)) v(t)$ and

$$
\left(r(t)\left(v^{\prime}(t)\right)^{\alpha}\right)^{\prime} \leq-k q(t)(1-p(\sigma(t)))^{\beta} v^{\beta}(\sigma(t))
$$

Since $\left(r\left(v^{\prime}\right)^{\alpha}\right)^{\prime}(t)<0$, we find

$$
v^{\prime}(\sigma(t)) \geq v^{\prime}(t)\left(\frac{r(t)}{r(\sigma(t))}\right)^{1 / \alpha}
$$

Define the function

$$
R(t)=\varphi(t) \frac{r(t)\left(v^{\prime}(t)\right)^{\alpha}}{v^{\alpha}(\sigma(t))}
$$

Hence, $R(t) \geq 0$. By differentiating $R(t)$ and using (2.39) and (2.40), we get

$$
R^{\prime}(t) \leq-\varphi(t) G(t)+\frac{\varphi^{\prime}(t)}{\varphi(t)} R(t)-\frac{\alpha \sigma^{\prime}(t)}{r^{1 / \alpha}(\sigma(t)) \varphi^{1 / \alpha}(t)} R^{1+1 / \alpha}(t) .
$$

Proceeding as in the proof of the previous case, we obtain

$$
\int_{t_{0}}^{t}\left(\varphi(v) G(v)-\frac{r(\sigma(v))\left(\varphi_{+}^{\prime}(v)\right)^{\alpha+1}}{(\alpha+1)^{\alpha+1}\left(\varphi(v) \sigma^{\prime}(v)\right)^{\alpha}}\right) \mathrm{d} v \leq R\left(t_{2}\right)
$$

which contradicts (2.33). This completes the proof.

Theorem 2.8 Assume that $\sigma^{\prime}(t)>0$. If there exist functions $\delta, \varphi \in C^{1}\left(\left[t_{0}, \infty\right),(0, \infty)\right)$ such that (2.33) holds and

$$
\limsup _{t \rightarrow \infty}\left(\frac{\pi^{\alpha}(t)}{\delta(t)} \int_{t}^{t}\left(k \delta(v) \eta(v) Q(v)-\frac{r(v)\left(\delta^{\prime}(v)\right)^{\alpha+1}}{(\alpha+1)^{\alpha+1} \delta^{\alpha}(v)}\right) \mathrm{d} v\right)>1
$$

for any $t \in\left[t_{0}, \infty\right)$, then (1.1) is oscillatory.

Proof Proceeding as in the proof of Theorem 2.7, we obtain that $v^{\prime}$ has one sign eventually. For the case where $v^{\prime}(t)<0$ for all $t \geq t_{1}$, let us define the function $\omega$ as in (2.34). From 
(2.35) we have

$$
\begin{aligned}
\omega^{\prime}(t)= & \frac{\delta^{\prime}(t)}{\delta(t)} \omega(t)+\delta(t) \frac{\left(r(t)\left(v^{\prime}(t)\right)^{\alpha}\right)^{\prime}}{v^{\alpha}(t)}-\frac{\alpha}{(\delta(t) r(t))^{\frac{1}{\alpha}}}\left(\omega(t)-\frac{\delta(t)}{\pi^{\alpha}(t)}\right)^{\frac{\alpha+1}{\alpha}} \\
& +\frac{\alpha \delta}{r^{\frac{1}{\alpha}}(t) \pi^{\alpha+1}(t)} .
\end{aligned}
$$

Using Lemma 1.1 with $C=\delta^{\prime}(t) / \delta(t), D=\alpha(\delta(t) r(t))^{\frac{-1}{\alpha}}$, and $V=\delta(t) / \pi^{\alpha}(t)$, we obtain

$$
\begin{aligned}
\omega^{\prime}(t) & \leq-k \delta(t) \eta(t) Q(t)+\frac{\delta^{\prime}(t)}{\pi^{\alpha}(t)}+\frac{r(t)\left(\delta^{\prime}(t)\right)^{\alpha+1}}{(\alpha+1)^{\alpha+1} \delta^{\alpha}(t)}+\frac{\alpha \delta}{r^{\frac{1}{\alpha}}(t) \pi^{\alpha+1}(t)} \\
& \leq-k \delta(t) \eta(t) Q(t)+\left(\frac{\delta(t)}{\pi^{\alpha}(t)}\right)^{\prime}+\frac{r(t)\left(\delta^{\prime}(t)\right)^{\alpha+1}}{(\alpha+1)^{\alpha+1} \delta^{\alpha}(t)} .
\end{aligned}
$$

Integrating (2.42) from $t_{2}$ to $t$, we get

$$
\begin{aligned}
& \int_{t_{2}}^{t}\left(k \delta(v) \eta(v) Q(v)-\frac{r(v)\left(\delta^{\prime}(v)\right)^{\alpha+1}}{(\alpha+1)^{\alpha+1} \delta^{\alpha}(v)}\right) \mathrm{d} v-\frac{\delta(t)}{\pi^{\alpha}(t)}+\frac{\delta\left(t_{2}\right)}{\pi^{\alpha}\left(t_{2}\right)} \\
& \quad \leq \omega\left(t_{2}\right)-\omega(t) .
\end{aligned}
$$

In view of the definition of $\omega(t)$, we get

$$
\begin{gathered}
\int_{t_{2}}^{t}\left(k \delta(v) \eta(v) Q(v)-\frac{r(v)\left(\delta^{\prime}(v)\right)^{\alpha+1}}{(\alpha+1)^{\alpha+1} \delta^{\alpha}(v)}\right) d v \\
\leq \delta\left(t_{2}\right) \frac{r\left(t_{2}\right)\left(v^{\prime}\left(t_{2}\right)\right)^{\alpha}}{v^{\alpha}\left(t_{2}\right)}-\delta(t) \frac{r(t)\left(v^{\prime}(t)\right)^{\alpha}}{v^{\alpha}(t)} .
\end{gathered}
$$

Therefore, from (2.8), it follows that

$$
\frac{r(t)\left(v^{\prime}(t)\right)^{\alpha}}{v^{\alpha}(t)} \geq-\frac{1}{\pi^{\alpha}(t)}
$$

Substituting the above inequality into (2.43), we are led to

$$
\frac{\pi^{\alpha}(t)}{\delta(t)} \int_{t_{2}}^{t}\left(k \delta(v) \eta(v) Q(v)-\frac{r(v)\left(\delta^{\prime}(v)\right)^{\alpha+1}}{(\alpha+1)^{\alpha+1} \delta^{\alpha}(v)}\right) \mathrm{d} v \leq 1 .
$$

Now, taking the $\limsup _{t \rightarrow \infty}$ on both sides of this inequality, we are led to contradiction.

On the other hand, let $v^{\prime}(t)>0$ for all $t \geq t_{1}$. The proof of this case is similar to that of Theorem 2.7, and so we omit it. Then the proof is complete.

Example 2.1 Consider the equation

$$
\left(t^{2 \alpha}\left[\left(u(t)+p_{0} u(\lambda t)\right)^{\prime}\right]^{\alpha}\right)^{\prime}+q_{0} t^{\gamma-1} u^{\beta}(\delta t)=0
$$

where $\alpha>0, \lambda, \delta \in(0,1), p_{0} \in[0, \lambda), q_{0}>0$, and $\gamma=\max \{\alpha, \beta\}$. We note that

$$
r(t):=t^{2 \alpha}, \quad p(t):=p_{0}, \quad \tau(t):=\lambda t, \quad \sigma(t):=\delta t, \quad q(t):=q_{0} t^{\gamma-1}
$$


and $f(u)=u^{\beta}$. It is easy to calculate that

$$
\pi(t)=\frac{1}{t} \quad \text { and } \quad Q(t)=q_{0}\left(1-\frac{p_{0}}{\lambda}\right)^{\beta} t^{\gamma-1} .
$$

From Theorem 2.3, equation (2.44) is oscillatory if the first order delay differential equation

$$
v^{\prime}(t)+\frac{K}{t^{2-\gamma / \alpha}} v^{\beta / \alpha}(\delta t)=0
$$

is oscillatory, where

$$
K=\left(\frac{q_{0}}{\gamma}\left(1-\frac{p_{0}}{\lambda}\right)^{\beta}\right)^{1 / \alpha}>0 .
$$

For $\alpha>\beta$, we see that $\gamma=\alpha$ and hence

$$
\int_{t_{0}}^{\infty} \frac{K}{t^{2-\gamma / \alpha}} \mathrm{d} v=\infty
$$

Then, by Corollary 2.2, equation (2.44) is oscillatory.

For $\alpha<\beta$, we have (2.13) holds if

$$
q_{0}\left(1-\frac{p_{0}}{\lambda}\right)^{\beta} a_{2}>\beta
$$

But this condition is not feasible as a result of constant $a_{2}$. However, according to Theorem 2.2, if we take $\gamma=\beta+1$, then (2.13) holds and hence equation (2.44) is oscillatory.

For $\alpha=\beta$, we have the following criteria for oscillation:

- By Theorem 2.2, we get the condition

$$
q_{0}\left(1-\frac{p_{0}}{\lambda}\right)^{\alpha}>\alpha
$$

- By Corollary 2.1, we get the condition

$$
q_{0}^{1 / \alpha}\left(1-\frac{p_{0}}{\lambda}\right) \ln \frac{1}{\delta}>\frac{\alpha^{1 / \alpha}}{\mathrm{e}}
$$

- By Theorem 2.6, we get the condition

$$
q_{0}\left(1-\frac{p_{0}}{\lambda}\right)\left(\frac{1}{\delta}\right)^{q_{0}\left(1-p_{0} / \lambda\right)}>1-q_{0}\left(1-\frac{p_{0}}{\lambda}\right) \quad \text { if } \alpha=1
$$

- By Theorem 2.8 with $\delta(t):=t^{-\alpha}$, we have that condition (2.41) holds if

$$
q_{0}\left(1-\frac{p_{0}}{\lambda}\right)^{\alpha}>\frac{\alpha^{\alpha+1}}{(\alpha+1)^{\alpha+1}}
$$


Table 1 Test of the strength of criteria for $\left(\mathrm{E}_{1}\right)$ and $\left(\mathrm{E}_{2}\right)$

\begin{tabular}{llll}
\hline & Condition $\left(\mathrm{C}_{1}\right)$ & Condition $\left(\mathrm{C}_{2}\right)$ & Condition $\left(\mathrm{C}_{4}\right)$ \\
\hline For $\left(\mathrm{E}_{1}\right)$ & $9_{0}>2.00000$ & $90>1.06150$ & $90>0.50000$ \\
For $\left(\mathrm{E}_{2}\right)$ & $9_{0}>1.25990$ & $90>0.18087$ & $90>0.19843$ \\
\hline
\end{tabular}

As special cases, we consider the equations

$$
\left(t^{2}\left[\left(u(t)+\frac{1}{3} u\left(\frac{2}{3} t\right)\right)^{\prime}\right]\right)^{\prime}+q_{0} u\left(\frac{1}{2} t\right)=0
$$

and

$$
\left(t^{2 / 3}\left[\left(u(t)+\frac{1}{3} u\left(\frac{2}{3} t\right)\right)^{\prime}\right]^{1 / 3}\right)^{\prime}+q_{0} u^{1 / 3}(0.01 t)=0
$$

From Table 1, we note that Condition $\left(\mathrm{C}_{4}\right)$ supports the most efficient condition for $\left(\mathrm{E}_{1}\right)$ and Condition $\left(\mathrm{C}_{2}\right)$ supports the most efficient condition for $\left(\mathrm{E}_{2}\right)$.

Moreover, for $\left(E_{1}\right)$, we see that Condition $\left(C_{3}\right)$ provides an improvement of Conditions $\left(\mathrm{C}_{1}\right)$ and $\left(\mathrm{C}_{2}\right)$, namely $q_{0}>0.8532$.

Also, for Euler differential equation, if $p_{0}=0$ and $\alpha=1$, then condition $\left(\mathrm{C}_{4}\right)$ reduces to $q_{0}>1 / 4$, which is sharp for oscillation.

\section{Acknowledgements}

The authors offer their earnest thanks to the editors and two anonymous referees.

\section{Funding}

The authors received no direct funding for this work.

\section{Availability of data and materials}

Data sharing not appropriate to this article as no datasets were produced down amid the current investigation.

\section{Competing interests}

The authors declare that they have no competing interests.

\section{Authors' contributions}

The authors contributed equally to the manuscript and read and approved the final manuscript.

\section{Publisher's Note}

Springer Nature remains neutral with regard to jurisdictional claims in published maps and institutional affiliations.

Received: 11 July 2019 Accepted: 4 March 2020 Published online: 14 March 2020

\section{References}

1. Agarwal, R.P., Grace, S.R., O'Regan, D.: Oscillation Theory for Second Order Linear, Half-Linear, Superlinear and Sublinear Dynamic Equations. Kluwer Academic, Dordrecht (2002)

2. Agarwal, R.P., Grace, S.R., O'Regan, D.: Oscillation Theory for Second Order Dynamic Equations. Series in Mathematical Analysis and Applications, vol. 5. Taylor \& Francis, London (2003)

3. Agarwal, R.P., Zhang, C., Li, T.: Some remarks on oscillation of second order neutral differential equations. Appl. Math. Comput. 274, 178-181 (2016)

4. Baculikova, B.: Oscillatory behavior of the second order functional differential equations. Appl. Math. Lett. 72, 35-41 (2017)

5. Bainov, D.D., Mishev, D.P.: Oscillation Theory for Neutral Differential Equations with Delay. Hilger, New York (1991)

6. Bazighifan, O., Elabbasy, E.M., Moaaz, O.: Oscillation of higher-order differential equations with distributed delay. J. Inequal. Appl. 2019, 55 (2019)

7. Bohner, M., Grace, S., Jadlovska, I.: Oscillation criteria for second-order neutral delay differential equations. Electron. J. Qual. Theory Differ. Equ. 2017,60 (2017)

8. Chatzarakis, G.E., Dzurina, J., Jadlovska, I.: New oscillation criteria for second-order half-linear advanced differential equations. Appl. Math. Comput. 347, 404-416 (2019)

9. Chatzarakis, G.E., Jadlovska, I.: Improved oscillation results for second-order half-linear delay differential equations, Hacet. J. Math. Stat. 48(1), 170-179 (2019) 
10. Dzurina, J., Jadlovska, I.: A note on oscillation of second-order delay differential equations. Appl. Math. Lett. 69, 126-132 (2017)

11. Elabbasy, E.M., Hassan, T.S., Moaaz, O.: Oscillation behavior of second order nonlinear neutral differential equations with deviating arguments. Opusc. Math. 32, 719-730 (2012)

12. Erbe, L.H., Kong, Q., Zhang, B.G.: Oscillation Theory for Functional Differential Equations. Dekker, New York (1995)

13. Grace, S.R., Dzurina, J., Jadlovska, I., Li, T.: An improved approach for studying oscillation of second-order neutral delay differential equations. J. Inequal. Appl. 2018, 193 (2018)

14. Hale, J.K.: Functional Differential Equations. Oxford Appl. Math. Sci., vol. 3. Springer, New York (1971)

15. Hale, J.K.: Theory of Functional Differential Equations. Springer, New York (1977)

16. Kitamura, Y., Kusano, T.: Oscillation of first-order nonlinear differential equations with deviating arguments. Proc. Am. Math. Soc. 78(1), 64-68 (1980)

17. Ladde, G.S., Lakshmikantham, V., Zhang, B.G.: Oscillation Theory of Differential Equations with Deviating Arguments. Dekker, New York (1987)

18. Liu, H., Meng, F., Liu, P.: Oscillation and asymptotic analysis on a new generalized Emden-Fowler equation. Appl. Math. Comput. 219, 2739-2748 (2012)

19. Luo, J.: Oscillation criteria for second-order quasi-linear neutral difference equations. Comput. Math. Appl. 43(12), 1549-1557 (2002)

20. Moaaz, O., Elabbasy, E.M., Bazighifan, O.: On the asymptotic behavior of fourth-order functional differential equations. Adv. Differ. Equ. 2017, 261 (2017)

21. Moaaz, O., Elabbasy, E.M., Muhib, A.: Oscillation criteria for even-order neutral differential equations with distributed deviating arguments. Adv. Differ. Equ. 2019, 297 (2019)

22. Philos, C.: On the existence of nonoscillatory solutions tending to zero at $\infty$ for differential equations with positive delays. Arch. Math. 36, 168-178 (1981)

23. Saker, S.: Oscillation Theory of Delay Differential and Difference Equations. VDM Verlag Dr. Muller, Saarbrucken (2010)

24. Sun, S., Li, T., Han, Z., Zhang, C.: On oscillation of second-order nonlinear neutral functional differential equations. Bull. Malays. Math. Sci. Soc. 36(3), 541-554 (2013)

25. Tang, X.H.: Oscillation for first order superlinear delay differential equations. J. Lond. Math. Soc. 65(2), 115-122 (2002)

26. $\mathrm{Wu}, \mathrm{H}$., Erbe, L., Peterson, A.: Oscillation of solution to second-order half-linear delay dynamic equations on time scales. Electron. J. Differ. Equ. 2016, 71 (2016)

27. $W u, Y ., Y u, Y .$, Xiao, J.: Oscillation of second-order Emden-Fowler neutral delay differential equations. Electron. J. Differ. Equ. 2018, 1 (2018)

28. Wu, Y., Yu, Y., Zhang, J., Xiao, J.: Oscillation criteria for second order Emden-Fowler functional differential equations of neutral type. J. Inequal. Appl. 2016, 328 (2016)

29. Zhang, C., Agarwal, R., Bohner, M., Li, T.: New results for oscillatory behavior of even-order half-linear delay differential equations. Appl. Math. Lett. 26, 179-183 (2013)

\section{Submit your manuscript to a SpringerOpen ${ }^{\circ}$ journal and benefit from:}

- Convenient online submission

- Rigorous peer review

- Open access: articles freely available online

- High visibility within the field

- Retaining the copyright to your article

Submit your next manuscript at $>$ springeropen.com 